\title{
Drug Resistance Pattern of the Isolated Organisms While Treating UTIs
}

\author{
Rozina Aktar Zahan ${ }^{1, ~}$, Nahreen Rahman ${ }^{1}$, Nilufar Yasmin², Md. Mostafizur Rahman ${ }^{3}$, \\ Parvez Hassan ${ }^{4}$ \\ ${ }^{1}$ Department of Microbiology, Rajshahi Medical College, Rajshahi, Bangladesh \\ ${ }^{2}$ Department of Virology, Rajshahi Medical College, Rajshahi, Bangladesh \\ ${ }^{3}$ Department of Skin and Venereology, Rajshahi Medical College, Rajshahi, Bangladesh \\ ${ }^{4}$ Institute of Biological Sciences, University of Rajshahi, Rajshahi, Bangladesh
}

\section{Email address:}

drrima14@gmail.com (R. A. Zahan)

${ }^{*}$ Corresponding author

\section{To cite this article:}

Rozina Aktar Zahan, Nahreen Rahman, Nilufar Yasmin, Md. Mostafizur Rahman, Parvez Hassan. Drug Resistance Pattern of the Isolated Organisms While Treating UTIs. American Journal of Laboratory Medicine. Vol. 5, No. 2, 2020, pp. 47-54.

doi: 10.11648/j.ajlm.20200502.12

Received: April 1, 2020; Accepted: May 6, 2020; Published: June 23, 2020

\begin{abstract}
Background: Urinary tract infections (UTI), which are caused by the presence and growth of microorganisms in the urinary tract, are perhaps the single commonest bacterial infections of mankind. Urinary tract infection is a most common infectious disease after respiratory tract infection in community practice. Aim of the study: The aim of this study is to assess the drug resistance pattern of the isolated organism while treating UTIs. Material \& Methods: This was a prospective study carried out in the Molecular Biology Laboratory, Institute of Biological Sciences, University of Rajshahi, Bangladesh during the period of July 2008 to June 2011. In the planning phase, we estimated a total sample size of 750 cases. The study population comprised of four hundred fifty (450) female patients clinically suspected of having UTI aged between 15-45 years attending the OPDs or admitted to Rajshahi Medical College Hospital, Rajshahi, SZMCH, Bogra, Combined Military Hospital (CMH), Bogra, Bangladesh, Rangpur Medical College, Rangpur, Dinajpur Medical College, Dinajpur in the Northern regions of Bangladesh. Selection of the participants of the study population was done on the basis of some inclusion and exclusion criteria. Results: The prevalence rate of urinary tract infection (UTI) of the present study population was therefore $33.55 \%$. Among the 151 confirm diagnosed UTI patients, asymptomatic UTI (Group A) was diagnosed in 54 women whereas, significant bacteriuria i.e. symptomatic UTI (Group B) was found in 97 patients. The prevalence of symptomatic UTI was therefore higher than asymptomatic UTI. The highest UTI patients 68 (44.44\%) were women within the 26-35 years age group followed by Group-C 46 (31.08\%) whose ages are within 36-45 years, while the Group-A women (15-25 years of age) were the least sufferers of UTI (24.83\%). The antibiotic susceptibility pattern of isolated uropathogens (both Gram-negative and Gram-positive bacteria) from the urine samples of the study population has been shown. The results showed that in general most of the urinary isolates showed higher resistance to commonly used and comparatively old drugs namely- Nalidixic acid, Cotrimoxazole, Nitrofurantoin, Ceftazidime, Ceftriaxone and Azithromycin. Cephradine showed moderate resistance (55\%). On the other hand, Staphylococcus saprophyticus was found to be highly sensitive to Imipenem (80\%), followed by gentamicin (71.4\%), Azithromycin (65.7\%), Amikacin (64.3\%). Ceftriaxone and Nirofurantoin both showed moderate sensitivity of $60 \%$. Staphylococcus aureus showed highest resistance to Ceftazidine (78\%), followed by Ciprofloxacin (77.5\%), Cotrimoxazole and Nalidixic acid (75\%), Ceftriaxone (66.7\%). Conclusion: The major pathogen E. Coli causing UTI in the Northern regions of Bangladesh and other gram negative (as well as gram positive) isolates were more highly sensitive to Imipenem, Amikacin and Gentamicin as compared to the other antibiotics tested.
\end{abstract}

Keywords: Urinary Tract Infections (UTI), Microorganism, Drug Resistance Pattern, E. coli 


\section{Introduction}

Urinary tract infections (UTI), which are caused by the presence and growth of microorganisms in the urinary tract, are perhaps the single commonest bacterial infections of mankind. [1] Urinary tract infection is a most common infectious disease after respiratory tract infection in community practice. [2] It remains a major public health problem in terms of morbidity and financial cost with an estimated 150 million cases per annum worldwide, [3-5] costing global economy in excess of 6 billion US dollars. [6] Nearly $10 \%$ of people will experience a UTI during their lifetime. [7, 8] Generally, Urinary tract infections (UTIs) are most common health problem for both sexes i.e. male and female. [9] Although UTIs occur in all age groups including men and women, clinical studies suggest that the overall prevalence of UTI is higher in women. UTIs are most commonly found in women of childbearing age and rarely occur in men. An estimated $50 \%$ of women experience at least one episode of UTI at some point of their lifetime and between $20 \%$ and $40 \%$ of women can have recurrent episodes within one year. $[10,11]$ One half of all women will experience a UTI in their lifetime, and one in three women will receive antimicrobial therapy for a UTI. In addition, the financial impact is enormous with costs exceeding \$1.6 billion for community acquired UTI. [12] Antimicrobial therapy is seldom indicated for the asymptomatic UTI; but it is usually indicated for symptomatic UTIs. [13] UTIs are often treated with different broad-spectrum antibiotics, one with a narrow spectrum of activity may be inappropriate because of emerging concerns about infection with resistant organisms. The most common antibiotics often used to treat routine, uncomplicated UTIs are Trimethoprim (sulfonamides), Trimethoprim/ Sulfamethoxazole, Cephalosporins, Fluoroquinolones (Ciprofloxacin, Ofloxacin, Norfloxacin, Trovafloxacin), Nitrofurantoin, Nalidixic acid, and Fosfomycin. [14] The emergence and spread of antibiotic resistance is a cause of increasing cancer. [5] Antibiotic resistance is the ability of a microorganism to withstand the effects of an antibiotic. It is one of the major causes of failure in the treatment of infectious diseases that results in increased morbidity, mortality, and costs of health care. [15] In the past decade, many kinds of resistant strains have been discovered. For example, Methicillin resistant Staphylococcus aureus (MRSA), [16] multidrug resistant Pseudomonas aeruginosa [17] and Serratia marcescens, [18] Vancomycin resistant Enterococci (VRE) [19] and extended spectrum beta lactamase (ESBL) resistant Enterococci. [20] Earlier reports in Bangladesh have shown on increased resistance of the urinary pathogens to commonly- used antibiotics. [21-23] Due to rising antibiotic resistance among uropathogens it is important to have local hospital-based knowledge of the organisms causing UTI and their antibiotic sensitivity patterns. This information would be relevant not only to the local hospital but would also be a vital regional database. Area-specific monitoring studies aimed to gain knowledge about the type of pathogens responsible for UTIs and their susceptibility patterns may help the clinicians to choose the right empirical treatment.

\section{Methodology and Materials}

This was a prospective study and was carried out in the Molecular Biology Laboratory, Institute of Biological Sciences, University of Rajshahi, Bangladesh during the period of July 2008 to June 2011, availing also some of the laboratory facilities of the Departments of Microbiology, Rajshahi Medical College Hospital, Rajshahi, SZMCH, Bogra, Combined Military Hospital, Bogra, Bangladesh, Rangpur Medical College Rangpur and Dinajpur Medical College Hospital, Dinajpur. We collected data from 450 patients with suspected UTI during the study. In the planning phase we estimated a total sample size of 750 cases. The study population comprised of four hundred fifty (450) female patients clinically suspected of having UTI aged between 15-45 years attending the OPDs or admitted to Rajshahi Medical College Hospital, Rajshahi, SZMCH, Bogra, Combined Military Hospital (CMH), Bogra, Bangladesh, Rangpur Medical College, Rangpur, Dinajpur Medical College, Dinajpur in the Northern regions of Bangladesh. Selection of the participants of the study population was done on the basis of some inclusion and exclusion criteria mentioned below.

1. Inclusion Criteria

a. Married and unmarried women of reproductive (child bearing age) i.e. 18-45 years of age.

b. Women having clinically suspected UTI. Clinical diagnostic criteria- dysuria, frequency, urgency and fever.

c. Women who are willing to participate in the study

2. Exclusion Criteria

a. Women below 18 and above 45 years of age and menopausal women.

b. Patients currently on antibiotic therapy or having history of receiving antibiotics within two weeks prior to enrolment in the study.

c. Women who are not willing to participate.

d. Patients on continuous indwelling catheter.

e. Women with severe concomitant diseases besides symptoms of UTI.

\section{Results}

Of the 450 patients screened for the presence of UTI, in the present study, one hundred fifty-one (151) patients were diagnosed of having UTI (both symptomatic and asymptomatic). The prevalence rate of urinary tract infection (UTI) of the present study population was therefore $33.55 \%$, as shown in Table 1. As shown in Table 1 , among the 151 confirm diagnosed UTI patients, asymptomatic UTI (Group A) was diagnosed in 54 women whereas, significant bacteriuria i.e. symptomatic UTI 
(Group B) was found in 97 patients (Table 1). The prevalence of symptomatic UTI was therefore higher than asymptomatic UTI. The distribution of asymptomatic UTI (Group A) and symptomatic UTI (Group B) subjects with respect to medico-demographic characteristics i.e. marital status (single, married, widow/divorced), pregnancy (pregnant, non-pregnant), diabetes (diabetic, non-diabetic) and blood pressure (hypotension, hypertension, normal blood pressure) has been presented in Table 1 . The incidence rate of UTI in relation to marital status, occurrence of pregnancy, presence of diabetes and high blood pressure has been graphically represented in the Figures $5 \mathrm{a}-5 \mathrm{~d}$. In the current study, only married women were considered for the pregnancy group and unmarried pregnant women were ruled out of the study because in our society there is no concept of such unmarried pregnant women and such cases, if exist, are not disclosed and thus are not available. The prevalence of UTI in relation to age of the subjects of the present study has been shown in Figure 1. The highest UTI patients 68 (44.44\%) were women within the 26-35 years age group (i.e. group-B women) followed by Group-C 46 (31.08\%) whose ages are within 36-45 years, while the Group-A women (15-25 years of age) were the least sufferers of UTI $(24.83 \%)$ i.e. 37 patients. The prevalence rate of UTI based on the educational qualifications of the respondents has been presented in Table 2. Education seems to play a significant role in preventing the incidence of UTI as patients having an educational qualification of Master's degree and above had a very low (4.64\%) incidence of UTI; while the incidence rate of UTI was very high (nearly $45.03 \%$ ) among the Illiterate. The frequency of occurrence of pathogens in urine samples of the study women has been shown in Table 3. Of the 151 isolates obtained, Gram-negative bacteria occurred more frequently than Gram-positive bacteria, constituting 99 $(65.56 \%)$ of the total isolates. Among the isolates, E. coli had the highest frequency of isolation with a frequency of 64 (42.38\%), followed by Pseudomonas aeruginosa 19 (12.58\%), Klebsiella spp. 8 (5.29\%) and Proteus sp also 8 (5.29\%). Gram-positive bacteria accounted for 40 (34.44\%) of the total isolates, with Staphylococcus saprophyticus 31 $(20.52 \%)$ and Staphylococcus aureus 9 (5.96\%). Mixed cultures of Klebsiella spp. and Staphylococcus spp. accounted for $12(7.947 \%)$ of the total isolated as shown in Table 4. Antibiotic susceptibility testing of the isolated uropathogens was performed by using the disc diffusion method described by Bauer et al. on Mueller Hinton Agar (Oxoid). The antibiotic susceptibility pattern of isolated uropathogens (both Gram-negative and Gram-positive bacteria) from the urine samples of the study population has been shown in Table 4. The results showed that in general most of the urinary isolates showed higher resistance to commonly used and comparatively old drugs namely- Nalidixic acid, Cotrimoxazole, Nitrofurantoin, Ceftazidime, Ceftriaxone and Azithromycin (Table 4, bar graph Figure 2 and Photographic Figure 4 showing very higher resistance (smaller zone of inhibition) by a urinary isolate. Ciprofloxacin and gatifloxacin exhibited moderate resistance and susceptibility (Photographic Figure 5 marked D and E). On the other hand, Imipenem, Gentamicin and Amikacin displayed very high sensitivity towards most of the isolated organism from urine samples (Table 4, bar graph Figure 2 and Photographic Figure 4 marked (A), (B) and (C). E. coli showed highest resistance to Azithromycin (85\%), followed by Nalidixic acid (77\%), Cotrimoxazole (68\%), Ceftazidine (63\%) and Ceftriaxone (60\%). Ciprofloxacin and Gatifloxacin with (55.56\%) and (48\%) resistance respectively were moderately resistant. On the other hand, $E$ coli showed highest sensitivity (91.2\%) to Imipenem, followed by Amikacin (83.55\%) and Gentamicin (78\%) sensitivity. Klebsiella showed highest resistance to Azithromycin (82\%), followed by Cefixime (78.5\%), Nalidixic acid (77.45\%), Gatifloxacin (77\%), Ceftriaxone (75.95\%), Ciprofloxacin (70\%) and Cotrimoxazole $(60.55 \%)$. On the other hand, Klebsiella was found to be highly sensitive towards Imipenem (92.08\%), Amikacin (91.5\%) and moderately sensitive to Cephradine (65.5\%). Proteus showed highest resistance to Nitrofurantoin (79.35\%), followed by Azithromicin (78\%), while Proteus showed moderate resistance of $55 \%$ to Nalidixic acid and Cotrimoxazole $(52.3 \%)$. On the other hand, Proteus was found to be highly sensitive to Amikacin (92.5\%), Ceftriaxone (89.17\%), Gentamicin (86.45\%), Gatifloxacin (75\%), Ceftazidine (72.55\%), Cirprofloxacin a cefixime and Cephradine (60\%). Pseudomonas showed highest resistance against Cephradine (90\%), followed by Gatifloxacin (85\%) and Azithromicin (85\%), Cefixime (82\%), Nalidixic acid resistance $(81.12 \%)$, Cotrimoxazole $(78.5 \%)$, Ceftazidine (75.5\%), Ceftriaxone (73.85\%). Pseudomonas was found to be highly sensitive towards Imipenem (94.5\%), Gentamicin (78.72\%), Amikacin (77\%) and moderately sensitive to Ciprofloxacin (55.32\%). Staphylococcus saprophyticus showed highest resistance to Nalidixic acid (78\%), Ceftazidine (77\%), Gatifloxacin (75.5\%), Cotrimoxazole (72\%), Ciprofloxacin (70\%). Cephradine showed moderate resistance (55\%). On the other hand, Staphylococcus saprophyticus was found to be highly sensitive to Imipenum (80\%), followed by Gentamicin (71.4\%), Azithromycin (65.7\%), Amikacin (64.3\%). Ceftriaxone and Nirofurantoin both showed moderate sensitivity of $60 \%$. Staphylococcus aureus showed highest resistance to Ceftazidine (78\%), followed by ciprofloxacin (77.5\%), Cotrimaxazole and Nalidixic acid (75\%), Ceftriaxone $(66.7 \%)$. On the contrary, Staphylococcus aureus showed highest sensitivity towards Imipenem (89\%), followed by Azithromycin (78\%), Gentamicin (75\%), Amikacin (71\%) and Gatifloxacin (70\%) while Cephradine showed $(65 \%)$ and Cefixime (60\%) sensitivity which can be considered as moderate sensitivity. 
Table 1. Distribution of asymptomatic UTI (Group A) and symptomatic UTI (Group-B) subjects with respect to medico-demographic characteristics ( $n=151)$.

\begin{tabular}{|c|c|c|c|c|c|c|}
\hline \multirow{2}{*}{ Characteristics } & \multicolumn{2}{|c|}{ Group A (Asymptomatic UTI) } & \multicolumn{2}{|c|}{ Group B (Symptomatic UTI) } & \multicolumn{2}{|c|}{ Total } \\
\hline & No. & $\%$ & No. & $\%$ & No. & $\%$ \\
\hline \multicolumn{7}{|l|}{ Marital status } \\
\hline Single & 12 & 22.22 & 37 & 38.14 & 49 & 32.45 \\
\hline Married & 38 & 70.37 & 57 & 58.76 & 95 & 62.91 \\
\hline Widow/divorced & 04 & 7.41 & 03 & 3.09 & 07 & 4.64 \\
\hline Total & 54 & 100 & 97 & 100 & 151 & 100 \\
\hline \multicolumn{7}{|l|}{ Pregnancy } \\
\hline Pregnant & 48 & 88.89 & 68 & 70.10 & 116 & 76.82 \\
\hline Total & 54 & 100 & 97 & 100 & 151 & 100 \\
\hline \multicolumn{7}{|l|}{ Diabetes } \\
\hline Non-diabetic & 22 & 40.74 & 36 & 37.11 & 58 & 38.41 \\
\hline Diabetic & 32 & 59.25 & 61 & 62.88 & 93 & 61.58 \\
\hline Total & 54 & 100 & 97 & 100 & 151 & 100 \\
\hline \multicolumn{7}{|l|}{ Blood Pressure (BP) } \\
\hline Normal BP & 23 & 42.59 & 36 & 37.11 & 59 & 39.07 \\
\hline Total & 54 & 100 & 97 & 100 & 151 & 100 \\
\hline
\end{tabular}

Table 2. The prevalence rate of UTI based on the educational qualifications of the women ( $n=151)$.

\begin{tabular}{ll}
\hline Educational Qualification & UTI Positive cases (\%) \\
\hline Illiterate & $68(45.03)$ \\
Primary & $38(25.17)$ \\
SSC & $24(15.89)$ \\
HSC/ Graduate & $14(9.27)$ \\
Masters and above & $7(4.64)$ \\
Total & $151(100 \%)$ \\
\hline
\end{tabular}

Table 3. Frequency of isolation of pathogens in urine samples of women. $(n=151)$.

\begin{tabular}{ll}
\hline Bacteria isolated & No. of isolates (\%) \\
\hline Gram-negative bacteria & $64(42.38)$ \\
Escherichia coli & $19(12.58)$ \\
Pseudomonas sp & $8(5.29)$ \\
Klebsiella sp. & $8(5.29)$ \\
Proteus sp. & $99(65.56 \%)$ \\
Total Gram -ive bacteria & \\
Gram-positive bacteria & $31(20.52)$ \\
Staphylococcus saprophyticus & $9(5.96)$ \\
Staphylococcus aureus & $40(26.49)$ \\
Total Gram + ive bacteria & $139(30.88)$ \\
Total Single bacterial growth (Gram + ive + Gram -ive) & $12(7.947)$ \\
Mixed bacterial growth of Klebsiella and Staphylococcus spp. & $151(100.0)$ \\
Total & \\
\hline
\end{tabular}

Table 4. Antimicrobial susceptibility (S) and resistance $(R)$ pattern of clinical bacterial strains isolated from UTI patients $(N=151)$.

\begin{tabular}{|c|c|c|c|c|c|c|c|}
\hline $\begin{array}{l}\text { Antimicrobial } \\
\text { agents }\end{array}$ & $\begin{array}{l}\text { Sensitivity } \\
\text { pattern }\end{array}$ & $\begin{array}{l}\text { E. coli } \\
n(\%)\end{array}$ & $\begin{array}{l}\text { Klebsiella } \\
\operatorname{spp} n(\%)\end{array}$ & $\begin{array}{l}\text { Proteus } \\
\operatorname{spp} n(\%)\end{array}$ & $\begin{array}{l}\text { Pseudomonas } \\
\operatorname{spp} n(\%)\end{array}$ & $\begin{array}{l}\text { S. saprophyticus } \\
\operatorname{spp} n(\%)\end{array}$ & $\begin{array}{l}\text { S. aureus } \\
\operatorname{spp} n(\%)\end{array}$ \\
\hline \multirow{2}{*}{ Cephradine } & $\mathrm{R}$ & 33.3 & 34.5 & 40 & 90 & 55 & 35 \\
\hline & $\mathrm{S}$ & 66.7 & 65.5 & 60 & 10 & 45 & 65 \\
\hline \multirow{2}{*}{ Cefixime } & $\mathrm{R}$ & 25 & 78.5 & 30 & 82 & 45 & 40 \\
\hline & $\mathrm{S}$ & 75 & 21.5 & 70 & 18 & 55 & 60 \\
\hline \multirow{2}{*}{ Ciprofloxacin } & $\mathrm{R}$ & 55.56 & 70 & 30 & 44.68 & 70 & 77.5 \\
\hline & $\mathrm{S}$ & 44.44 & 30 & 70 & 55.32 & 30 & 22.5 \\
\hline \multirow{2}{*}{ Gatifloxacin } & $\mathrm{R}$ & 48 & 77 & 25 & 85 & 75.5 & 30 \\
\hline & $\mathrm{S}$ & 52 & 23 & 75 & 15 & 24.5 & 70 \\
\hline TMP/SMZ & $\mathrm{R}$ & 68 & 60.55 & 52.3 & 78.5 & 72 & 75 \\
\hline Cotrimoxazole & $\mathrm{S}$ & 32 & 39.45 & 47.7 & 21.5 & 28 & 25 \\
\hline \multirow{2}{*}{ Nitrofurantoin } & $\mathrm{R}$ & 34 & 41.67 & 79.35 & 80 & 40 & 45 \\
\hline & $\mathrm{S}$ & 66 & 58.33 & 20.65 & 20 & 60 & 55 \\
\hline Nalidixic Acid & $\mathrm{R}$ & 77 & 77.45 & 55 & 81.12 & 78 & 75 \\
\hline
\end{tabular}




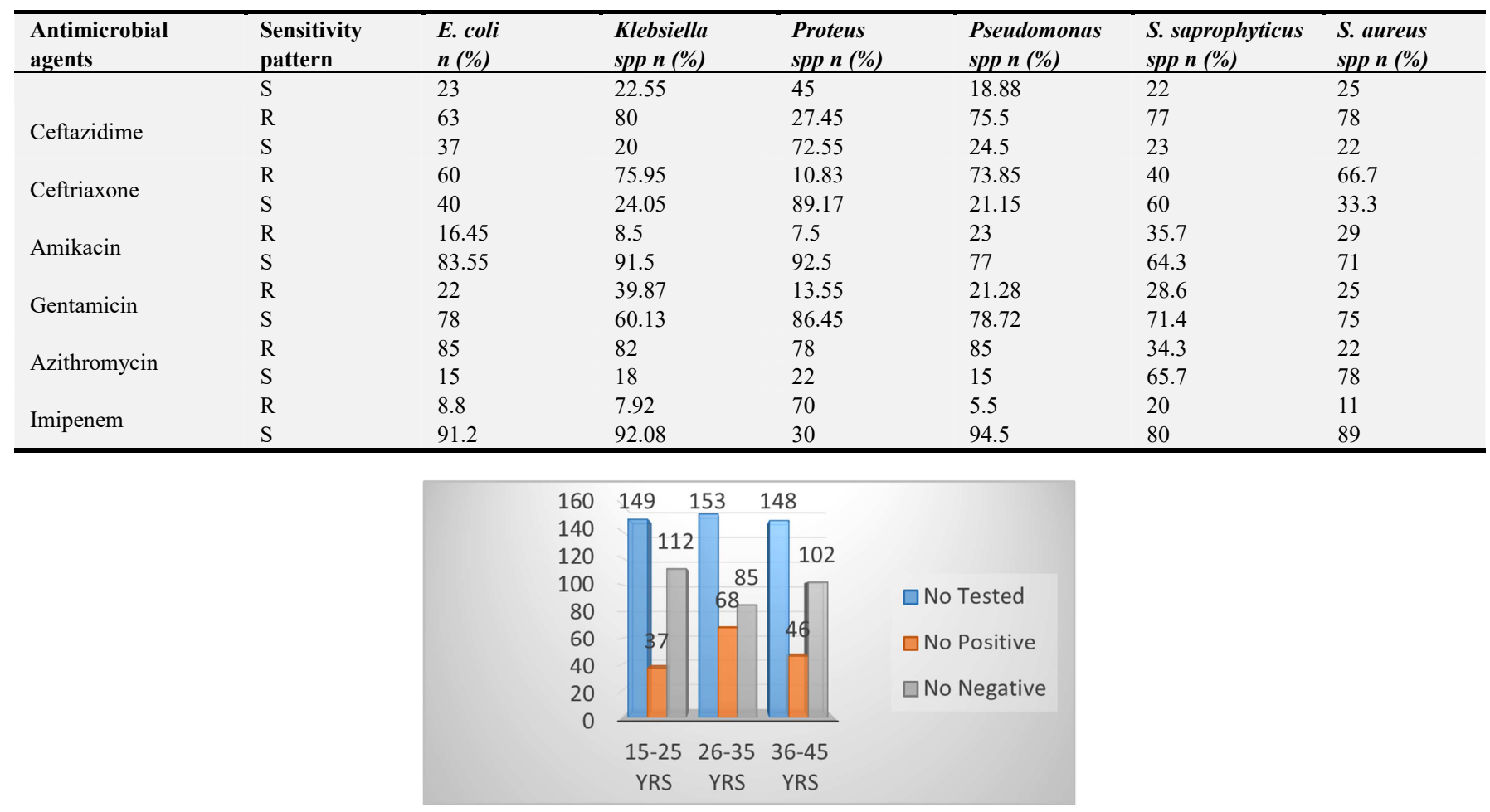

Figure 1. Age-wise distribution of women of the study population $(n=450)$.

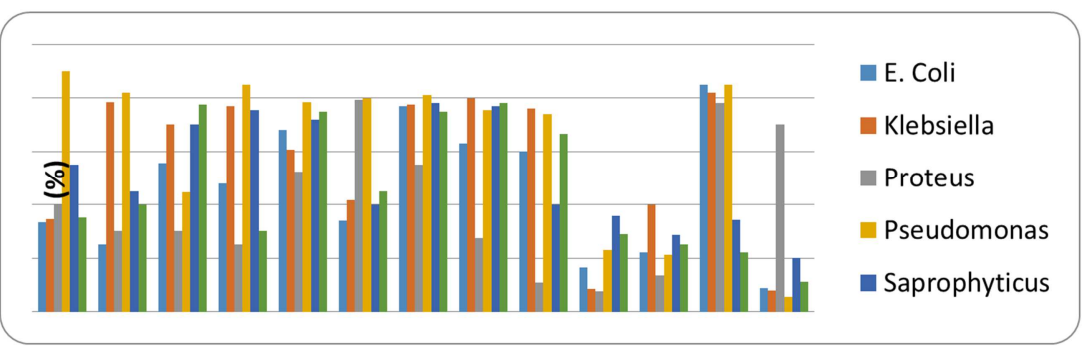

Figure 2. Antibiotic resistance pattern of the bacterial species isolated from urine samples.

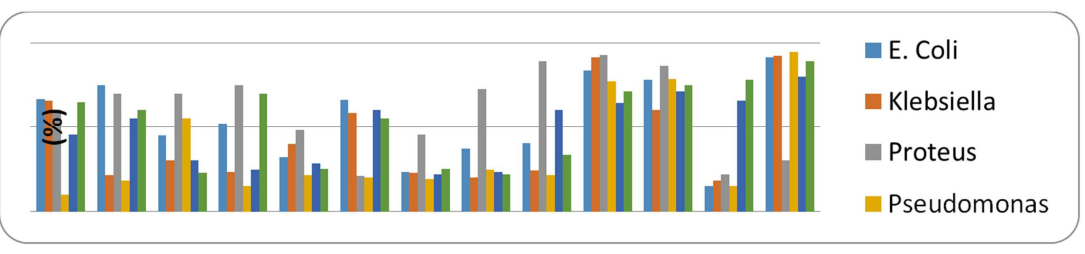

Figure 3. Antibiotic sensitivity pattern of the bacterial species isolated from urine samples.

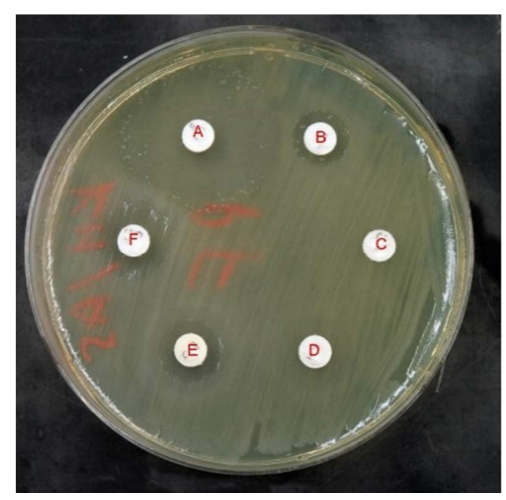

Figure 4. Azithromycin in antibiotic susceptibility testing by disc diffusion method.

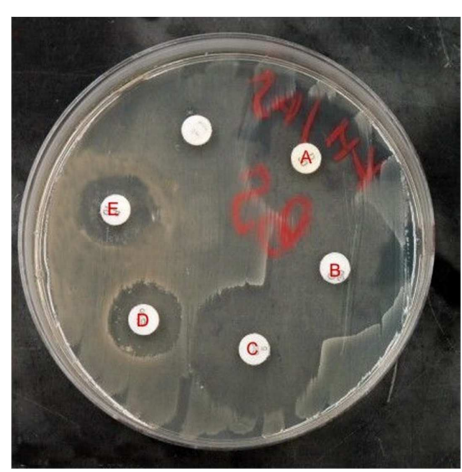

Figure 5. Sensitivity of Imipenem (A), Gentamicin (B), Amikacin (C), Ciprofloxacin (D) and Gatifloxacin (E) exhibited moderate resistance and moderate susceptibility. 


\section{Discussion}

In the present study, midstream urine samples were collected from female patients having clinically suspected UTI attending the OPDs or admitted to Rajshahi Medical College Hospital, Rajshahi, SZMCH, Bogra, Rangpur Medical College, Rangpur, Dinajpur Medical College, Dinajpur, Combined Military Hospital (CMH), Bogra located in the Northern regions of Bangladesh. In the present study, the predominance of both asymptomatic and symptomatic UTIs among pregnant women (88.89 vs. $70.10 \%)$ was noticed as compared to non-pregnant $(29.63 \%$ vs. $29.90 \%)$. The urinary tract undergoes profound physiological and anatomical changes during pregnancy facilitating the development of bacteriuria both symptomatic and asymptomatic in women. [24] Diabetes mellitus (DM) has long been considered to be a predisposing factor for urinary tract infection [25] and the urinary tract is the principle site of the infection in diabetics with increased risk of complications of UTI. [26] It is evident from literature that diabetic subjects are at high risk of UTIs. For instance, in a study [27], the prevalence of UTI in diabetic subjects were found to be higher when compared with non-diabetic subjects ( $9 \%$ vs. $0.78 \%$ ). Symptomatic and asymptomatic UTIs occur more frequently in women with diabetes mellitus than women without diabetes mellitus. [28] However, gestational diabetes mellitus was not associated with increased risk of UTls. [29] Observation of the current study is that symptomatic and asymptomatic UTIs were more common in hypertensive subjects $(59.79 \%$ vs. $55.55 \%)$ than subjects with normal blood pressure $(37.11 \%$ vs. $42.59 \%)$ and hypotension $(3.09 \%$ vs. $1.85 \%)$. Study of age-wise incidence of UTI showed that the highest UTI sufferers (44.44\%) were the most sexually active women (26-35 years age group) followed by $(31.08 \%)$ of $36-45$ years; while the least sufferers of UTI $(24.83 \%)$ were women of $15-25$ years. A study conducted in Bangladesh by Kawser Parveen et al. [30] reported high incidence of UTI in 21-25 years age group $(44.61 \%)$ and show disagreement with our finding. Education seems to play a significant role in preventing the incidence of UTI and its incidence was extremely low (4.64\%) in patients having Master's degree; while very high (45.03\%) among the Illiterate in the present study. This is consistent with many studies, which shows that UTI is more prevalent among young married women. This finding of ours shows good harmony with another similar study conducted in our country by Kawser Parveen et al. [30] In their study, the significance of education was also evidenced by the fact that only $10 \%$ of the patients suffering from bacteriuria were educated while $90 \%$ were illiterate. Of the 151 isolates analyzed, Gramnegative bacteria occurred more frequently than Grampositive bacteria, constituting $99(65.56 \%)$ of the total isolates. Among the Gram (-)ve organisms the significant isolate was $E$. coli which had the highest percentage of isolation 64 (42.38\%), followed by Pseudomonas aeruginosa 19 (12.58\%), Klebsiella spp. 8 (5.29\%) and Proteus sp 8 (5.29\%). Gram-positive bacteria accounted for 40 (34.44\%) of the total isolates, with Staphylococcus saprophyticus 31 (20.52\%) and Staphylococcus aureus 9 (5.96\%). Mixed cultures of Klebsiella spp. and Staphylococcus spp. accounted for $12(7.947 \%)$ of the total isolates. The most common bacterial isolates from midstream urine samples of women from asymptomatic UTI and symptomatic UTI enrolled in our study were Gram - negative Escherichia coli (42.38\%), ) followed by Pseudomonas aeruginosa (12.58\%). Rahman et al. [31] and Ahmed and Rashid [32] in their studies in Bangladesh also reported $E$. coli as being the commonest pathogen responsible for bacteriuria which is consistent with the findings of this study. The findings of $E$. coli are also in agreement with the study done by Sharmin [33] in Bangladesh. Another study done by Hasan et al. [34] in a tertiary hospital in Indian study showed $50.7 \%$ incidence of UTI caused by $E$. coli, which was nearer to our study. UTI caused by $P$. aeruginosa isolated were $10.78 \%$ reported by Sharmin [33] in Bangladesh. Anbumani and Mallika [36] showed $11 \%$ UTI caused by $P$. aeruginosa were very close to our finding. As per findings of our study, $E$ coli exhibited highest sensitivity $(91.2 \%)$ towards Imipenem, followed by Amikacin (83.55\%) and Gentamicin (78\%) sensitivity (Table 4) which is in good agreement with the same findings by Sharmin [34] in Bangladesh. Our study found Klebsiella showing highest resistance to Azithromycin (82\%), followed by Cefixime (78.5\%), Nalidixic acid $(77.45 \%)$, Gatifloxacin (77\%), Ceftriaxone (75.95\%), Ciprofloxacin (70\%) and Cotrimoxazole $(60.55 \%)$. In our study similarly, Klebsiella was found to be highly sensitive towards Imipenem (92.08\%), Amikacin (91.5\%). Farzana Rahman et al. [36] in their study in Dhaka City reported Klebsiella spp. also showed high sensitivity to Amikacin (91.5\%) and Imipenem $(92.08 \%)$ which is in harmony with our findings. In our study Proteus species showed highest resistance to Nitrofurantoin (79.35\%), followed by Azithromicin (78\%). Study done in India showed almost similar results observed by us where Proteus was $100 \%$ resistant towards Nitrofurantoin. [35] Kenechukwu et al. [37] also reported in another study that Proteus was $70 \%$ resistant to Nitrofurantoin shows good harmony with our study findings. In our study Proteus showed moderate resistance of $55 \%$ to Nalidixic acid and Cotrimoxazole $(52.3 \%)$. High sensitivity was also observed to Gentamicin $(86.20 \%)$ and Ceftriaxone $(72.41 \%)$, which is consistent with our finding.

\section{Limitations of the Study}

In spite of maximum sincerity and dedication invested to carry out the present study; it is never free of limitations as the sample size was not large enough. Again, due to lack of proper logistic support, the genetic analysis of resistant bacteria that could help us finding the actual cause behind the emerging drug resistance could not be performed by us.

\section{Conclusion and Recommendations}

Under the present study, very alarming level of antibiotic 
resistance has been observed were Ciprofloxacin and even newer floroquinones like Gatifloxacin, the broad-spectrum antibiotics and major anti-pseudomonad weapons are becoming moderately sensitive to bacteria causing UTI. Ciprofloxacin, Gatifloxacin, Cephradine and Cefixime (Except Klebsiella and Pseudomonas showing > 79 and 90\% resistance, respectively) exhibited moderate to less moderate sensitivity in many cases under the study. The overall antibiotic susceptibility testing of the major isolated uropathogen $E$. coli. and other uropathogens of the present study indicated that most uropathogens exhibited very higher level of resistance to the commonly used antibiotics such as Azithromycin, Nalidixic acid and Cotrimoxazole. These drugs have limited value for the treatment of UTI and should no longer be used. Moreover, from the findings of the study it can be concluded that the major pathogen E. coli causing UTI in the Northern regions of Bangladesh and other gram negative (as well as gram positive) isolates were more highly sensitive to Imipenem, Amikacin and Gentamicin as compared to the other antibiotics tested. It is recommended that, antibiotics should be prescribed after performing a routine microscopy and culture/ sensitivity of urine in order to inhibit acquisition and spread of drug resistance by the bacteria. Antimicrobial sensitivity testing is needed for selection of antibiotics for treatment of UTI patients. Routine monitoring of drug resistance pattern will help to identify the resistance trends regionally.

\section{Funding Source}

Self

\section{Conflict of Interest}

Not declared

\section{Declaration}

Dr. Rozina Aktar Zahan is also a PhD Fellow, Institute of Biological Sciences, University of Rajshahi, Rajshahi, Bangladesh

\section{References}

[1] Theodore M. 2007. Prevalence and antibiogram of urinary tract infections among prison inmates in Nigeria. The Internet Journal of Microbiology 3 (2), 12-23.

[2] Hryniewicz K, Szczypa K, Sulilowska A, Jankowski K, Betlejewska K, Hryniewicz W. 2001. Antibiotic susceptibility of bacterial strains isolated from urinary tract infections in Poland. J Antimicrob Chemother 47 (6), 773-780.

[3] Stamm WE, Norrby SR. 2001. Urinary tract infections: disease panorama and challenges. Journal of Infectious Diseases 183 (1), S1-S4.

[4] Fakhrossadat M, Narges S. 2009. Changing patterns in sensitivity of bacterial uropathogens to antibiotics in children.
Pakistan Journal of Medical Sciences 25 (5), 801-805.

[5] Gupta K. 2001. Increasing antimicrobial resistance and the management of uncomplicated community-acquired urinary tract infections. Int J Antimicrob Agents 135, 41-50.

[6] Foxman B. 2003. Epidemiology of urinary tract infections: Incidence, morbidity, economic costs. Disease a Month 49, 53-70.

[7] Hoberman A, Wald ER. 1997. Urinary tract infections in young febrile children. Pediatr Infect Dis J 16, 11-17.

[8] Delanghe J, Kouri TT, Huber AR, Hannemann-Pohl, K, Guder WG, Lun A. 2000. The role of automated urine particle flow cytometry in clinical practice. Clin Chim Acta 301, 1-18.

[9] Kristen ML. 2004. Management of urinary tract infection during pregnancy. American J Matern Child Nursing 29 (4), 254-258.

[10] Siiri K, Kai T, Inga V, Jelena S, Epp S, Marika M. 2009. Persistence of Escherichia coli clones and phenotypic and genotypic antibiotic resistance in recurrent urinary tract infection in childhood. J Clin Microbiol 47, 99-105.

[11] Vasquez V, Hand WL. 2004. Antibiotic susceptibility patterns of community-acquired urinary tract infection isolates from female patients on the US (Texas)-Mexico border. J Appl Res 4 (2), 321-326.

[12] Nicoll LE. 2003. Urinary tract infection: Traditional pharmacological therapies. Dis Mon 49 (2), 1128.

[13] Prais D, Straussbcrg R, Avitzur Y, Nussinovitch M, Harei U, Amir J. 2003. Bacterial susceptibility to oral antibiotics in community acquired urinary tract infection. Archives of Disease in Childhood 88, 215-218.

[14] Bouza E, Cercenado E. 2002. Klebsiella and Enterobacter: Antibiotic resistance and treatment implications. Semin Respir Infect 17, 215-230.

[15] Wagenlehner FM, Naber KG. 2004. New drugs for grampositive uropathogens. Int J Antimicrob Agents 24, S39-S43.

[16] Linuma Y. 2007. Infection control strategies for antimicrobial resistance. Nippon Rinsho 65, 175-184.

[17] Kim BN, Choi SI, Ryoo NH. 2006. three-year follow-up of an outbreak of Serratia marcescens bacteriuria in a neurosurgical intensive care unit. J Korean Med Sci 21, 973-978.

[18] Gold HS. 2001. Vancomycin-resistant enterococci: Mechanisms and clinical observations. Clin Infect Dis 33, 210-219.

[19] Bhattacharya S. (2006). ESBL: from Petri dish to the patient. Indian J Med Microbiol 24, 20-24.

[20] Khatun AK, Rashid H, Chowdhury TA. 1985. Prevalence of urinary tract infection in pregnancy. J Bangladesh Coll Phys Surg 2, 6-10.

[21] Khaleque SA, Islam KMN, Ishaq M, Kamal N. 1990. Pattern of bacterial growth of 3022 urine samples cultured in a private laboratory. Bangladesh Private Medical practitioners Journal 1, 9-13.

[22] Chowdhury MZ, Muhammad F, Rahman MAK, Ahmed AA. 1994. Bacterial etiology and sensitivity pattern of UTI cases in Sher-E-Bangla Medical College, Barishal. Journal of Preventive and Social Medicine 13, 62-65. 
[23] Bhowmick BK, Rashid H. 2004. Prevalence and antibiotic susceptibility of E. coli isolated from urinary tract infection (UTI) in Bangladesh. Pakistan Journal of Biological Sciences 7 (5), 717- 720 .

[24] Begum N. 1992. Clinical profile of urinary tract infection in pregnancy. Mymensingh Med J 1, 6-10.

[25] Bonadio M, Costarelli S, Morelli G, Tartaglia T. 2006. The influence of diabetes mellitus on the spectrum of uropathogens and the antimicrobial resistance in elderly adult patients with urinary tract infection. BMC Infect Dis 6, 54 .

[26] Sahib AKY. 2008. Study of ciprofloxacin resistant Escherichia coli (CREC) in type 2 diabetic patients with symptomatic urinary tract infections. Iraq J Comm Med 21 (1), 58-63.

[27] Goswami R, Bal CS, Tejaswi S, Punjabi GV, Kapu A, Kochupiilai N. 2001. Prevalence of urinary tract infection and renal scars in patients with diabetes mellitus. Diabetes Research and Clinical Practice 53, 181-186.

[28] Daneshgari F, Mooree C. 2006. Diebetic uropathy. Semin Nephroly 26 (2), 182-185.

[29] Rizk DE, Musta N, Thomas L. 2001. The prevalence of urinary tract infections in patients with gestational diabetes mellitus. Int Vrogynecol J Pelvic Floor Dysfunc 12 (5), 317322.

[30] Kawser Parveen, Afroza Momen, Arzumath Ara Begum, Monowara Begum. 2011. Prevalence of Urinary Tract
Infection during Pregnancy. J Dhaka National Med Coll Hos $17(02), 8-12$.

[31] Rahman T, Haque F, Begum J, Khan IH. 1990. Urinary tract infection in diabetic and non-diabetic patients. Bangladesh Renal J 9, 8-12.

[32] Ahmed S, Rashid HU. 1996. Urinary tract infection in adults: A review. Bangladesh Renal J 15, 23-31.

[33] Sharmin S. 2005. Use of chromogenic media (Urochrom II) for detection of uropathogen. M. Phil (Microbiology) Thesis; University of Dhaka. pp 55-68.

[34] Hasan AS, Nair D, Kaur J, Baweja G, Deb M, Agarwal P. 2007. Resistance pattern of Urinary isolates in a tertiary Indian hospital. Journal of Ayub Medical College Abbattabad 19 (1), 39-41.

[35] Anbumani N, Mallika M. 2007. Antibiotic Resistance Pattern in Uropathogens in a Tertiary Care Hospital. Indian Journal for the Practising Doctor 4 (1), 23-25.

[36] Farjana Rahman, Sadia Chowdhury, Md. Majibur Rahman, Dilruba Ahmed, Anowar Hossain. Antimicrobial Resistance Pattern of Gram-negative Bacteria Causing Urinary Tract Infection. 2009. S. J. Pharm. Sci. 2 (1): 44-50.

[37] Kenechukwu M, Chinekwu O, Davidson N, Golibe UO. 2005. Antibiotic Sensitivity Patterns in Urinary Tract Infections at a Tertiary Hospital. Journal of the University of Nigeria Medical Students 1, 1-5. 